\title{
Exploration of double clad fibers for increased stability of bidirectional free space optical links
}

Sarah A. Tedder, Bryan L. Schoenholz, Joel Berkson, Bertram Floyd

Sarah A. Tedder, Bryan L. Schoenholz, Joel Berkson, Bertram Floyd, "Exploration of double clad fibers for increased stability of bidirectional free space optical links," Proc. SPIE 10524, Free-Space Laser Communication and Atmospheric Propagation XXX, 105240X (15 February 2018); doi: $10.1117 / 12.2287428$

SPIE. Event: SPIE LASE, 2018, San Francisco, California, United States 


\title{
Exploration of Double Clad Fibers for Increased Stability of Bidirectional Free Space Optical Links
}

\author{
Sarah A. Tedder*a, Bryan L. Schoenholz ${ }^{\mathrm{a}}$, Joel Berkson ${ }^{\mathrm{b}}$, and Bertram Floyd ${ }^{\mathrm{c}}$ \\ aNASA Glenn Research Center, 2100 Brookpark Rd, Cleveland, OH, USA 44135-3127; \\ 'The University of Arizona, Tuscan, Arizona, USA, 85721; \\ ${ }^{c}$ Sierra Lobo, 2100 Brookpark Rd, Cleveland, OH, USA 44135-3127;
}

\begin{abstract}
Bidirectional, high data rate, low size, weight, and power (SWaP), and low cost free space optical links are needed for space and aeronautic communication applications to send and receive large volumes of data. We are exploring design strategies for optical transceivers to reduce $\mathrm{SWaP}$ and cost through increased misalignment tolerance (pointing requirement reduction) and sharing the optical transmit and receive paths (imposing optical symmetry). In applications where the detector is fiber coupled, the receive fiber numerical aperture is the main driver of the pointing accuracy requirement. Increasing the numerical aperture of the receive fiber reduces the pointing requirement. In an optically symmetric design, the fibers both transmit and receive the light. Hence, increasing the receive fiber numerical aperture requires a similar increase of the transmit fiber. Unfortunately, increasing the transmit fiber numerical aperture causes instability in received power over small misalignments. Double clad fibers offer a solution. These fibers transmit from a single mode core and receive light in a larger numerical aperture. Results show that as transceiver fibers, double clad fibers have an improved misalignment tolerance and a higher stability for small changes in misalignment when compared to single mode fibers and multimode fibers. Also, double clad fibers are shown to match the performance of an asymmetrical link design with a single mode transmit fiber and a multimode receive fiber.
\end{abstract}

Keywords: Double clad fiber, pointing accuracy, free space optical link.

\section{INTRODUCTION}

High data rate $(\sim 10 \mathrm{~Gb} / \mathrm{s})$, low size, weight, and power (SWaP), and low cost free space optical links (FSOLs) are needed for space and aeronautical communication applications to send and receive large volumes of science data. Previously, we explored ways to reduce SWaP and cost for an Intra International Space Station optical link ${ }^{1}$. In Ref. [1], we examined the design strategies: passive misalignment tolerance (reducing the pointing accuracy requirement), sharing the optical transmit and receive paths (imposing optical symmetry), and using small form factor pluggable (SFP) optical transceivers. In this paper we explore double clad fiber's (DCF) capability to implement all of these design strategies.

One of the greatest challenges of free space optical links is pointing the laser beam with enough accuracy to create a high speed link ${ }^{2}$. This pointing accuracy challenge is commonly solved by using active components such as gimbals, motorized mounts, and adaptive optics ${ }^{2,3}$. These solutions can be costly and increase the system's SWaP. In applications where the laser and detector are fiber coupled, the pointing accuracy requirement is driven by the numerical aperture of the receive fiber. To increase the pointing accuracy tolerance, a solution is an asymmetrical design of the transmitter and receiver ${ }^{4}$. An asymmetrical design allows the receive fiber numerical aperture to be increased while preserving the transmitted beam quality. In these systems, the light is transmitted through a single mode fiber, producing a near diffraction limited Gaussian beam, and received through a large numerical aperture fiber.

In this paper we look to incorporate a large core receive fiber in a system with one common transmit and receive path. Sharing the optical paths enables the further reduction of SWaP by reducing the amount of optics needed for bidirectional communication. Our previous study ${ }^{1}$ showed that increasing the transmitter and receiver core fiber size increased the pointing accuracy tolerance. However, the increased core size reduced the received power stability under small alignment changes. This power instability was attributed to the transmitted power profile from the larger core fiber. This paper shows that DCFs can increase the pointing accuracy tolerance while improving the received power

*sarah.a.tedder@nasa.gov; phone 1216 433-6591; nasa.gov

Free-Space Laser Communication and Atmospheric Propagation XXX, edited by Hamid Hemmati,

Don M. Boroson, Proc. of SPIE Vol. 10524, 105240X · (c) 2018 SPIE

CCC code: $0277-786 X / 18 / \$ 18 \cdot$ doi: $10.1117 / 12.2287428$

Proc. of SPIE Vol. 10524 105240X-1 
stability. This is because a DCF can transmit through a single mode fiber core and receive light through a multimode cladding within the same cable and using the same optical axis ${ }^{5}$.

This paper will compare measured pointing accuracy tolerance and received power stability of DCFs to single mode and multimode fibers in a 20 meter free space optical link. The results show that as transceiver fibers, DCFs have better performance than single mode and multimode fiber. DCFs will be shown to match the performance of an asymmetrical system with a single mode transmit fiber and multimode receive fiber.

\section{EXPERIMENTAL SETUP}

The data is collected using a free-space optical link setup on two optical tables placed 20 meters apart. Pointing accuracy tolerances are measured by misaligning the optics laterally. The transmitter is mounted on a vertical motorized stage and the receiver is mounted on a horizontal motorized stage, as seen in Fig. 1. A $1550 \mathrm{~nm}$ laser is created by a $10 \mathrm{~Gb} / \mathrm{s} \mathrm{SFP+}$ emitting near $2 \mathrm{dBm}$ of power. A fiber optic cable under test sends the light to an aspheric lens with a numerical aperture of 0.25 and a focal length of $11 \mathrm{~mm}$. The light then propagates 20 meters where it is collected by another identical lens and then sent to the receive test fiber. The received light is measured using a power meter. The power meter has an InGaAs photodiode fiber sensor, which has a power range of $100 \mathrm{pW}-3 \mathrm{~mW}$ and a measurement uncertainty of $\pm 5 \%$. A beam profiler was used to measure the power profile directly after the transmitting lens. The profiler is also an InGaAs based detector with a pixel size of $4.54 \mu \mathrm{m}$ and area of $12.5 \mathrm{~mm}$ by $10 \mathrm{~mm}$. Environmental conditions were monitored with a tiltmeter. The tiltmeter measured ambient temperature and the angle of the transmit optical table in 2 directions. These 2 directions were aligned parallel and perpendicular to the optical path on the surface of the optical table. The tiltmeter has a $0.1 \mu$ radians angle resolution and $0.1{ }^{\circ} \mathrm{C}$ temperature resolution.

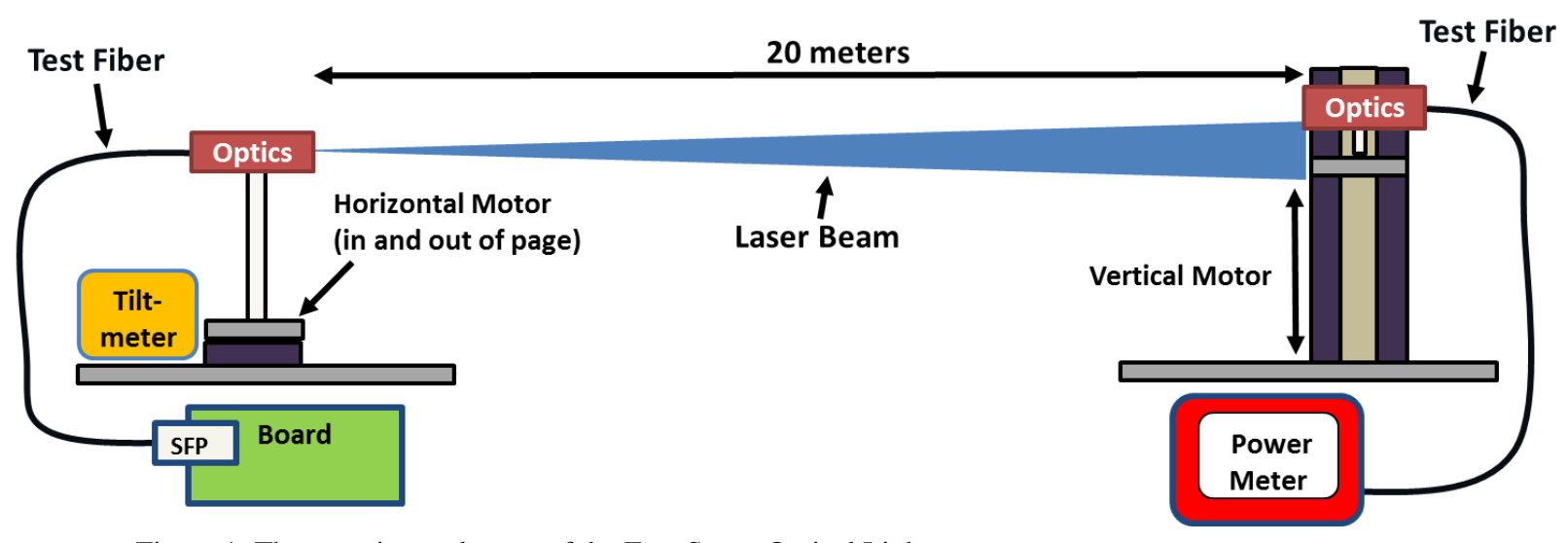

Figure 1: The experimental setup of the Free Space Optical Link system

\subsection{Test Fibers}

Test fibers were used for transmitting the light from the SFP+ to the lens and collecting the light at the receiver, as shown in Fig.1. Each test fiber is 2 meters long. Information about each fiber tested is given in Table 1 below. These fibers were chosen to evaluate the performance of DCFs. Having a single core and two claddings, DCF's are capable of guiding light through both the core as well as the first layer of cladding. We selected a DCF with a single mode core to transmit a Gaussian beam profile. This single mode inner core has a numerical aperture of 0.12 , a core diameter of 9 $\mu \mathrm{m}$, and a mode field diameter of $\sim 10.4 \mu \mathrm{m}$. It was shown in our previous work ${ }^{1}$ that the larger the receive core fiber the larger the pointing accuracy tolerance. Therefore, we selected the first cladding to be $105 \mu \mathrm{m}$ in diameter - the largest diameter that could be made by the manufacturer that is compatible with the LC (Lucent Connector) connection needed by the SFPs. The first cladding has a numerical aperture of 0.2. The second type of test fiber used is a single mode fiber (SMF) which is a standard industry fiber used typically in terrestrial fiber networking applications. The SMF was chosen to compare to the DCF because it has a matching diameter with the DCF inner core. The final test fiber used is a multimode fiber (MMF) with a core size to match the first cladding size of the DCF. The numerical aperture of the DCF first cladding almost matches as it is only 0.02 smaller than the MMF. 
Table 1: Test Fibers

\begin{tabular}{llll}
\hline \hline Fiber & Core Size, $\boldsymbol{\mu m}$ & $\begin{array}{l}\text { Graded Index } \\
\text { or Step Index }\end{array}$ & $\begin{array}{l}\text { Numerical } \\
\text { Aperture }\end{array}$ \\
\hline Single Mode Fiber (SMF) & $10.4^{\mathrm{a}}$ & Graded & $0.12^{\mathrm{b}}$ \\
Multimode Fiber (MMF) & 105 & Step & 0.22 \\
Double Clad Fiber (DCF) & 9,105 & Step & $0.12,0.2$ \\
\hline \hline
\end{tabular}

${ }^{a}$ Mode Field Diameter. ${ }^{b}$ Not Given, Typical Reported

\subsection{Pointing Accuracy Data Collection}

We define pointing accuracy tolerance as the amount of lateral misalignment between the transmitter and receiver before the received power is below the detector receive limit. Two-axis power profile measurements are collected using motorized stages to decenter the transmit and receive optics. A program is used to adjust the vertical and horizontal stages in a grid around the center while reading and recording received power. A power profile is collected at a range of divergence angles to identify the maximum pointing accuracy tolerance for each fiber. The divergence angle was adjusted by changing the distance from the fiber to the lens using a stepper motor. The transmit and receive fiber to lens distance was always adjusted symmetrically. The test fibers' pointing accuracy tolerances were measured over a range of detector minimum receive limits.

The horizontal motorized stage has a travel range of $220 \mathrm{~mm}$, an absolute on-axis accuracy of $\pm 2.0 \mu \mathrm{m}$, a home location accuracy of $\pm 0.25 \mu \mathrm{m}$, and a minimum incremental movement of $0.1 \mu \mathrm{m}$. The vertical motorized stage has a travel range of $300 \mathrm{~mm}$, a calibrated on-axis accuracy less than $\pm 5.0 \mu \mathrm{m}$, a home location accuracy of $\pm 0.6 \mu \mathrm{m}$, and a minimum achievable incremental movement of $100 \mathrm{~nm}$. The typical data set used incremental movements of 3 or $5 \mathrm{~mm}$ to create the grid of data.

\subsection{Power Stability Data Collection}

Effects of small changes in alignment on the power were collected with the power meter and tiltmeter. The optics were aligned for maximum power throughput at the beginning of each test with the divergence angle set to collimated. Data was then collected with a computer program that alternates samples from the power meter and a tiltmeter every 3 seconds for 10 hours. While the data was collected, the ambient room temperature varied causing changes in the mechanical components of the system. Changes in mechanical components caused optical misalignment. One of these changes is the angle of the optical tables. The angle of the transmit optical table was recorded with the tiltmeter.

\section{DATA ANAYLSIS}

\subsection{Pointing Accuracy Tolerance}

The collected 2-axis power profiles are analyzed to determine the pointing accuracy tolerance. The pointing accuracy tolerance is found by taking cross sections of the 2-axis profile at the maximum power location in both the horizontal and vertical directions. The distance over which the received power is above each detector receive limit is then identified for both the horizontal and vertical directions. The distances measured for the horizontal and vertical directions are compared and the minimum is reported as the lateral misalignment tolerance (pointing accuracy tolerance). The larger the lateral misalignment tolerance, the lower the required pointing accuracy.

\subsection{Power Stability}

The power measurements are converted to a power change by taking the difference between each measurement and the measured maximum power. The ambient temperature at which the maximum power occurs is considered the optimum 
temperature. This optimum temperature is considered to have a zero power change, and all other temperature are measured relative to the optimum temperature. The average and standard deviation of power change are found for each 0.1 degree Celsius increment over the full range in temperature change

\section{THEORETICAL MODEL}

Zemax controlled with Python was used to find the theoretical pointing accuracy tolerance of the 20 meter FSOL. Python is used to iterate Zemax through divergence angles and off center positions of the receiver. This is done in order to find the optimum divergence angle that gives the maximum pointing accuracy tolerance based on the power coupled into the receive fiber. The theoretical model currently only models lasers transmitted through SMFs.

At a distance of 20 meters, diffraction effects are significant, so Gaussian beam theory is used as much as possible for increased accuracy. A Gaussian propagation model is used through the transmitter over the 20 meters and stops at the receiver. At the receiver, the Gaussian beam equations no longer have closed form solutions because they must pass through an aperture off-center. For this reason, a hybrid of the ray-trace approach and the beam propagation approach is used.

Through the off centered system, we emulate the beam by creating an equivalent ray field. Using the beam's radius of curvature and the beam size just before the receiver lens, a point source is created. This point source is placed at the distance of the radius of curvature from the first surface of the receiver lens with a matching numerical aperture. The power density distribution of the beam is defined by using an apodized Gaussian beam profile. Zemax defines their Gaussian intensity distribution function type using the apodization factor below:

$$
A(\rho)=\exp \left(-G \rho^{2}\right)
$$

where $A$ is the amplitude, $G$ is the apodization factor, and $\rho$ is the normalized pupil radius. The definition of apodization factor is:

$$
G=\left(\frac{E P D}{\omega}\right)^{2}
$$

where EPD is the exit pupil diameter, and $\omega$ is the beam size. With the correct beam apodization and beam parameters defined at the receiver lens, rays are traced to the receive fiber aperture. The ray-trace model is then used to determine the amount of energy coupled into the receive fiber.

\section{RESULTS AND DISCUSSION}

Double clad fiber performance in a 20 meter FSOL with $\sim 2 \mathrm{dBm}$ transmitted power is compared to SMF and MMF fiber performance in the same system. Test results are presented and discussed in this section. First the pointing accuracy tolerance comparison is presented. Next the receiver power stability of the system under small changes in alignment is presented.

\subsection{Pointing Accuracy Tolerance}

DCF pointing accuracy tolerance is compared to SMF and $105 \mu \mathrm{m}$ MMF by measuring the lateral misalignment tolerance of the 20 meter link. The maximum allowable lateral tolerance was measured at the optimum divergence angle for a range of detector receive limits. Figure 2 shows a plot of the maximum lateral misalignment tolerance verses the detector receive limit for each fiber combination measured. This plot shows that for all fibers the lateral misalignment tolerance decreases as the detector receive limit increases. The measured results are shown as symbols and the lines show the theoretical model predictions. The theoretical model for SMF transmitting to MMF (dashed line) matches well to the measured results (triangles). The SMF theory (solid line) doesn't match as well to its measured results (circles). This may be caused by the difficulty to achieve perfect alignment of the optics because of SMF's small alignment tolerance. Other possibilities are the over estimation of captured rays in the theoretical model. The model of the receiver is done by ray tracing which doesn't included diffraction effects. 


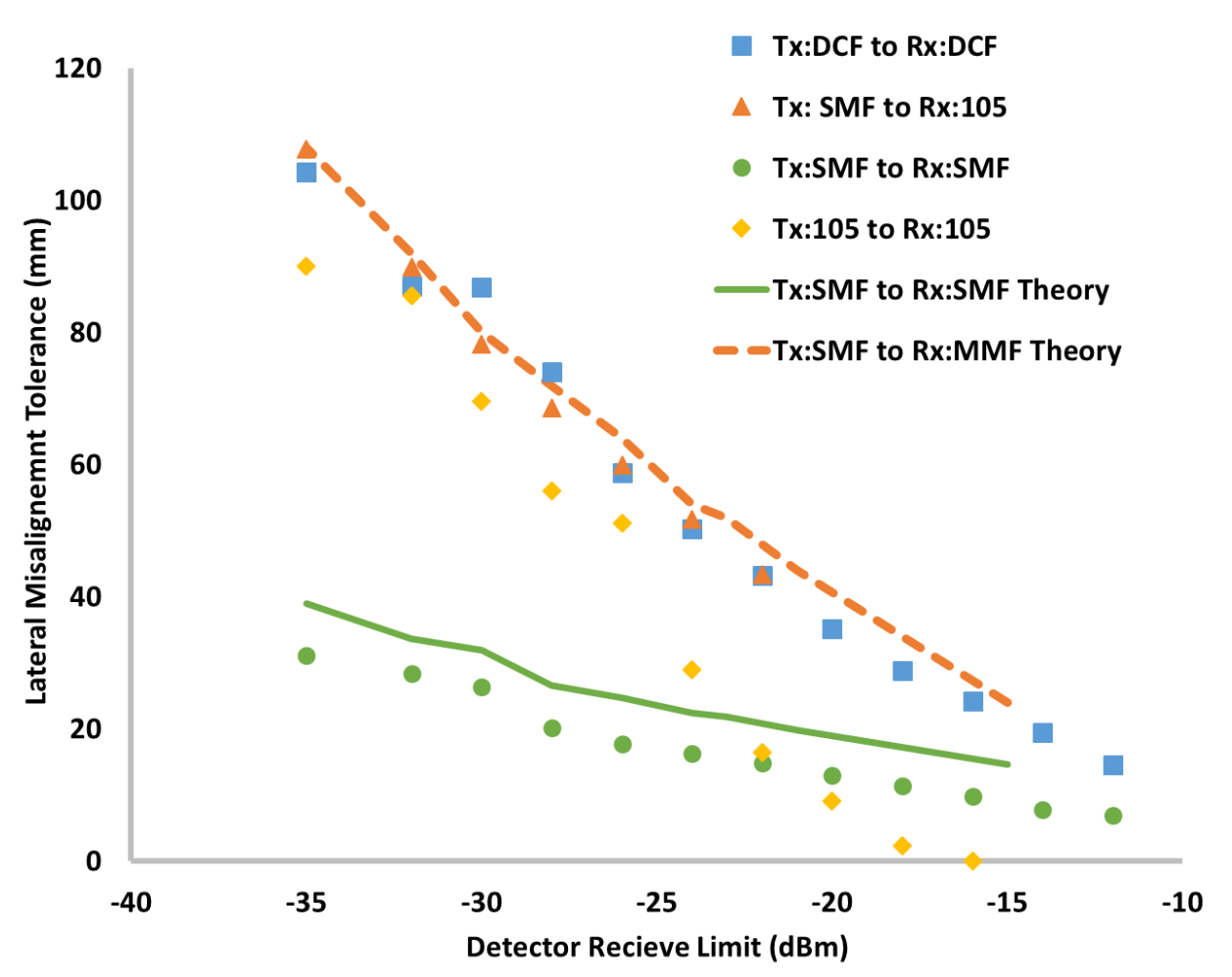

Figure 2: Plot of the maximum lateral misalignment tolerance verses the detector receive limit for a variety of fibers used in a 20 meter FSOL with $\sim 2 \mathrm{dBm}$ transmitted power. Symbols show measured results, lines represent theoretical model predictions.

In Fig. 2, a number of important features can be pointed out. DCFs (squares) have an almost identical performance to SMF transmitting to the MMF fiber. The SMF to SMF misalignment tolerance is smaller than the DCF over the full range of measured receive limits. The MMF to MMF (diamonds) results have a smaller tolerance than the SMF to SMF at detector receive limits higher than $-22 \mathrm{dBm}$. At receive limits lower than $-22 \mathrm{dBm}$ the MMF approaches the performance of the DCF. In the region from $-26 \mathrm{dBm}$ to $-35 \mathrm{dBm}$ the ratio of the misalignment tolerance of both DCF and MMF to SMF is constant. Within this detector limit range DCF lateral misalignment tolerance is $3.3 \pm 0.2$ times larger than SMF, whereas the MMF is only $2.6 \pm 0.45$ times larger. In summary, these results show that the DCF performs the same as a SMF transmitting to a MMF and better than a MMF transmitting to MMF. This indicates that DCFs enable an optically symmetric FSOL design with a higher pointing accuracy tolerance.

\subsection{Power Stability under Small Alignment Changes}

The received power stability of DCF transmitting to DCF under small alignment changes is compared to other fiber configurations in Figure 3. These small alignment changes are plotted in terms of ambient temperature changes that caused the system to misalign. Changes in temperature and power are referenced from the optimum system alignment with the transmitted beam collimated. The change in received power is averaged over each 0.1 degree Celsius incremental change. The error bars represent the standard deviation of the received power change over each 0.1 degree temperature change. For all fibers the average power change increases with increasing temperature change, since changes in temperatures cause changes in the mechanical components of the system. One of these changes is the angle of the optical tables. Figure 4 shows the change in the angle of the transmit optical table in the optical axis direction verses temperature change. Both the angle change in Fig. 4 and the power change in Fig. 3 increase with temperature change. This shows the connection between temperature change and optical misalignment. The standard deviation of the angle change is larger than the average angle change over the full temperature range. This large standard deviation indicates that temperature may not be the only factor effecting the angle of the tables. 


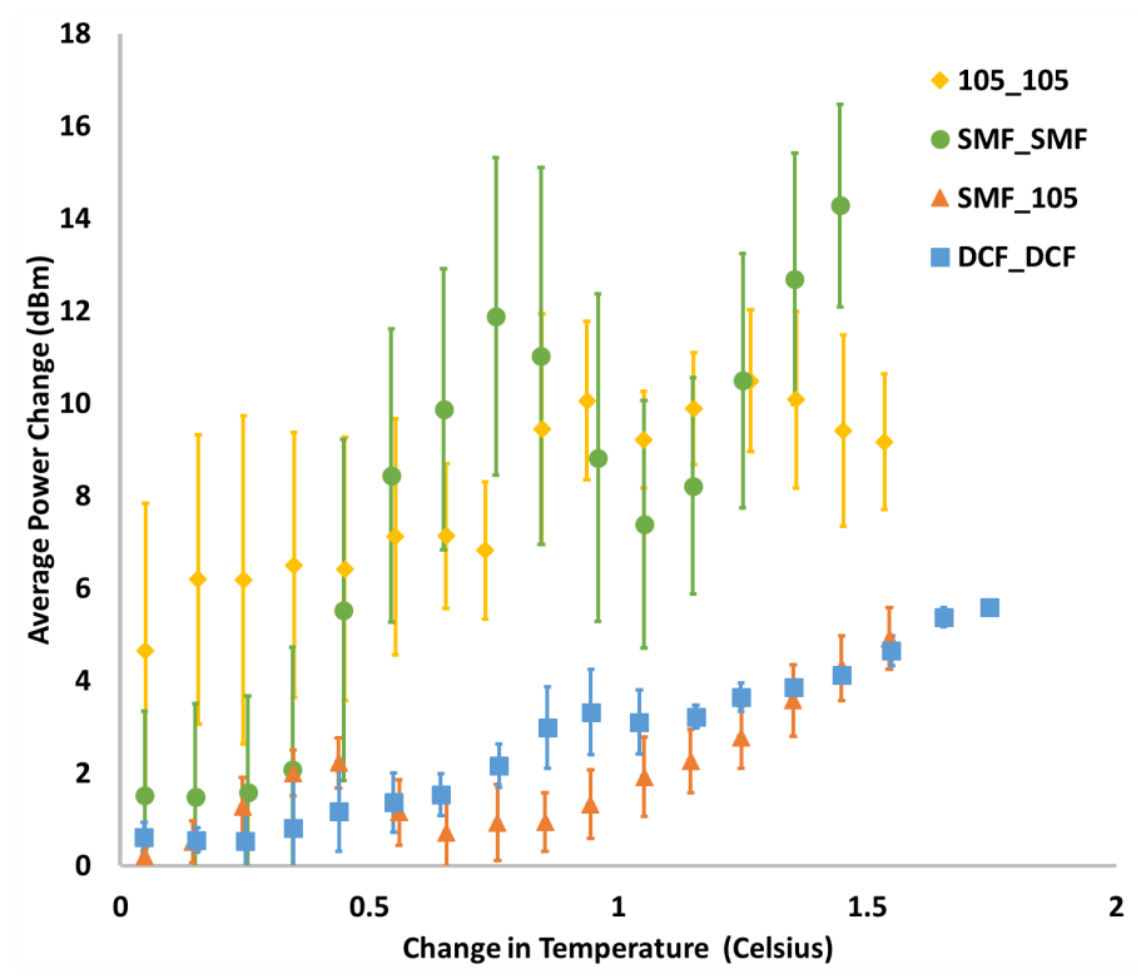

Figure 3: The angle change in the optical axis averaged over $0.1^{\circ} \mathrm{C}$ plotted over the temperature range.

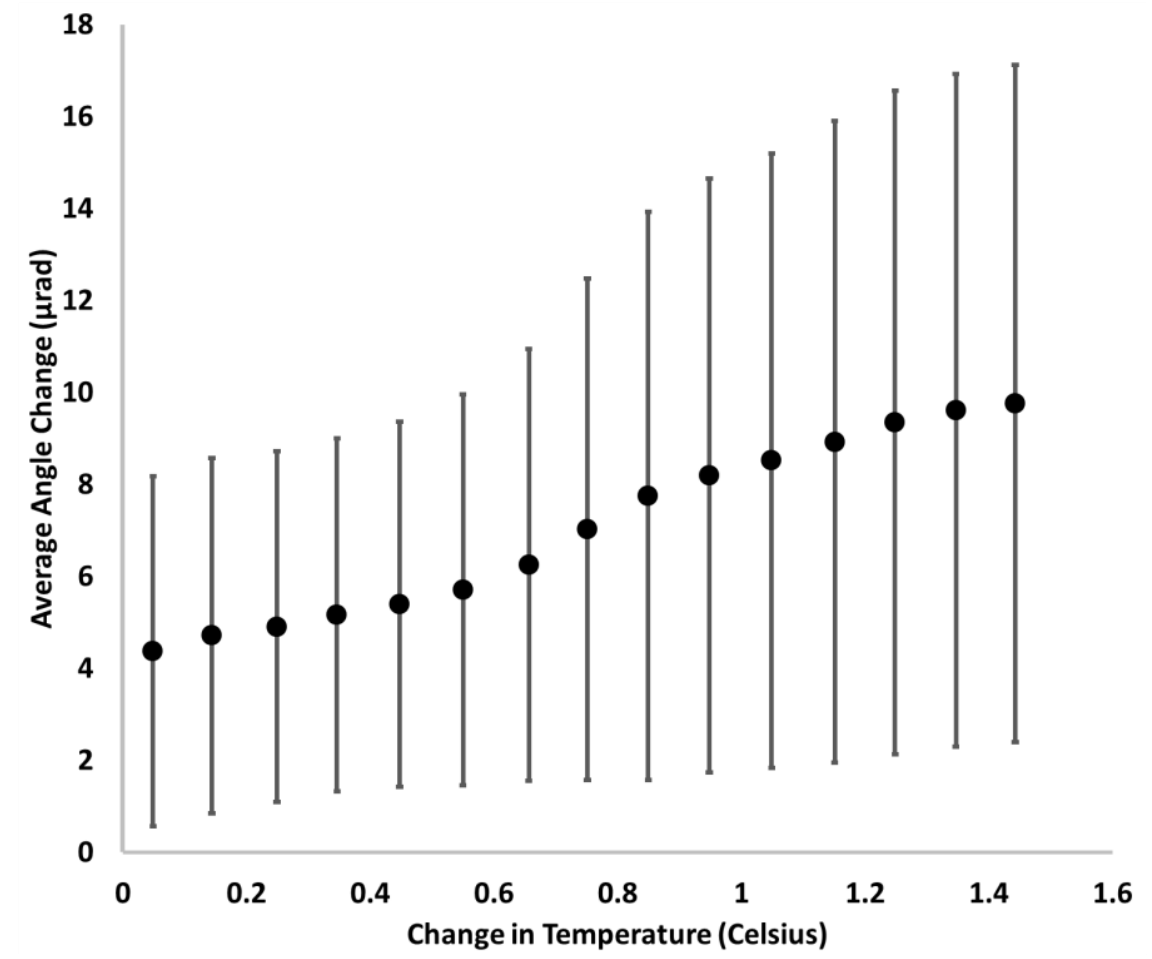

Figure 4: Plot of the power change verses temperature. Power change measurements are averaged over 0.1 degrees Celsius. Error bars represent the standard deviation over the 0.1 degree change. 
a)

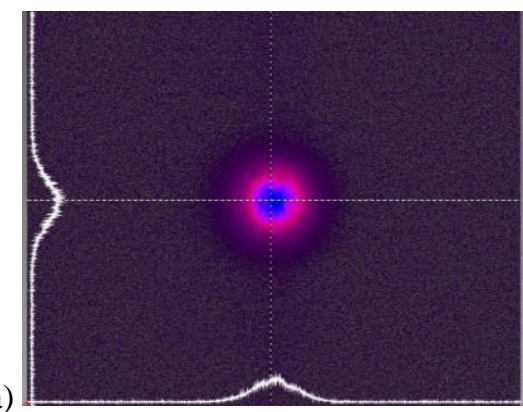

b)
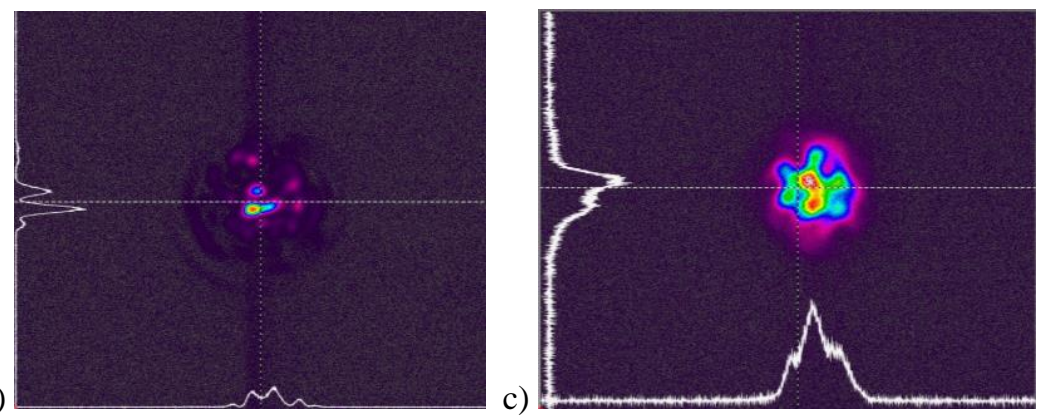

Figure 5: Transmitted beam power profiles. a) single mode fiber, b) multimode fiber, c) double clad fiber.

Fig. 3 shows that the DCF (squares) has the same received power stability as a SMF transmitting to a MMF with a 105 $\mu \mathrm{m}$ core (triangles). Both show an average of $\sim 5 \mathrm{~dB}$ less change in power than MMF to MMF (diamonds) over the full range of temperature change. These results agree with our previous work ${ }^{1}$ where we showed that increasing the transmit fiber core size decreases the received power stability. The SMF to SMF (circles) has average power changes that are similar to the DCF at temperature changes less than $0.4^{\circ} \mathrm{C}$. At temperature changes larger than $0.5^{\circ} \mathrm{C}$, the SMF to SMF average power change increases to more than the MMF to MMF. This can be attributed to the small numerical aperture of the SMF.

Beam profiles were collected to investigate the power instabilities relation to the transmitted power profile. These profiles were collected immediately after exiting the transmit lens. Figure 5 a) shows the transmitted power profile of the SMF, b) is the MMF, and c) is the DCF. The modes of the MMF cause more spatial variance of the power than the SMF and DCF. This spatial variance causes areas of low power. Therefore small changes in angle of the transmitter can change the power profile from a peak to a valley.

\section{CONCLUSION}

In summary, DCFs match the pointing accuracy tolerance and received power stability of a SMF transmitting to a MMF of the same core/first cladding size. But unlike SMF to MMF where the optical link design is asymmetric requiring two optical paths for bidirectional communication, DCFs may enable a symmetrical design. To verify this, bit error rate testing needs to be completed. Also the received power stability presented was collected at a collimated divergence angle. This would not be the divergence angle at which a 20 meter FSOL would operate. Instead the optimum divergence angle would be used. These results and the bit error rate will be presented in a future publication.

In conclusion, DCF have the potential to replace 2 fibers (transmit and receive with different core sizes) with one fiber. This enables bidirectional FSOL designs with one optical path and no active control, resulting in lower SWaP. DCFs should be considered as solution for high cost SWaP applications where detectors and transmitting sources are fiber coupled.

\section{ACKNOWLEDGEMENTS}

This work is supported by the Tech and Standards Division within NASA Space Communications and Navigation $(\mathrm{SCaN})$ Program and the Communications \& Intelligent Systems Division. We want to thank Patrick Millican for helping with initial laser alignment.

\section{REFERENCES}

[1] Tedder, S. A., Scheonholz, B. L., and Suddath, S., "Study of Lateral Misalignment Tolerance of a Symmetric Free Space Optical Link for the Development of an Intra ISS Point to Point Wireless Optical Link", 34th AIAA International Communications Satellite Systems Conference, Cleveland, Ohio, October, 2016.

[2] Hemmati, H., [Near-Earth Laser Communications], CRC Press, Boca, Florida, 2009. 
[3] Hemmati, H, [Deep Space optical Communication], John Wiley and Sons, Inc. Hoboken, New Jersey, 2006.

[4] Grein, M. E. et al, "Design of a Ground-Based Optical Receiver for the Lunar Laser Communications Demonstration", International Conference on Space Optical Systems and Applications, 2011.

[5] Brunetti, A. C., Margulis, W., and Rottwitt, K, "Raman probes based on optically-poled double-clad fiber and coupler", Optics Express, Vol. 20, No. 27, Dec. 2012. 